\title{
Application of MALDI-TOF MS to assess clinical characteristics, risk factors, and outcomes associated with anaerobic bloodstream infection: a retrospective observational study
}

Tsuyoshi Watanabe ${ }^{1 *} \mathbb{0}$, Yuki Hara², Yusuke Yoshimi ${ }^{3}$, Waka Yokoyama-kokuryo ${ }^{1}$, Yoshiro Fujita1,4, Masamichi Yokoe ${ }^{3}$ and Yoshinori Noguchi ${ }^{3}$

\begin{abstract}
Background: Correctly identifying anaerobic bloodstream infections (BSIs) is difficult. However, a new technique, matrix-assisted laser desorption ionization-time of flight mass spectrometry (MALDI-TOF MS), enables more accurate identification and appropriate treatment. Anaerobic BSIs identified by MALDI-TOF MS were retrospectively analyzed to determine the clinical and microbiological features and patient outcomes based on the anaerobic genera or group.

Methods: Medical records of patients with anaerobic BSIs were used to conduct a single-center retrospective cohort study from January 2016 to December 2020 in Nagoya, Japan. Multivariate logistic regression analysis was performed to determine the independent risk factors for in-hospital mortality.

Results: Of the 215 patients with anaerobic BSIs, 31 had multiple anaerobic organisms in the blood culture, including 264 total episodes of anaerobic BSIs. Bacteroides spp. were isolated the most $(n=74)$, followed by gram-positive non-spore-forming bacilli $(n=57)$, Clostridium spp. $(n=52)$, gram-positive anaerobic cocci (GPAC) $(n=27)$, and gramnegative cocci $(n=7)$. The median patient age was 76 years; $56.7 \%$ were male. The most common focal infection site was intra-abdominal (36.7\%). The in-hospital mortality caused by anaerobic BSIs was $21.3 \%$, and was highest with Clostridium spp. (36.5\%) and lowest with GPAC (3.7\%). Age, solid tumors, and Clostridium spp. were independent risk factors for in-hospital mortality.
\end{abstract}

Conclusions: We identified current anaerobic BSI trends using MALDI-TOF MS and reported that mortality in patients with anaerobic BSIs patients was highest with Clostridium spp. infections.

Keywords: Anaerobic blood stream infection (BSI), Matrix-assisted laser desorption ionization-time of flight mass spectrometry (MALDI-TOF MS), Clostridium species (spp.)

*Correspondence: tsuyoshiwatanaberhythm@yahoo.co.jp

1 Division of Rheumatology, Chubu Rosai Hospital, 2-10-15, Komei-cho, Minato-ku, Nagoya, Aichi 455-8530, Japan

Full list of author information is available at the end of the article

\section{Background}

Anaerobic microorganisms are major components of the mucosal membrane microflora in humans, with several hundred species identified by classical molecular methods. Anaerobic bloodstream infections (BSIs) are implicated in $4-10.4 \%$ of bacteremic episodes with a high

c) The Author(s) 2021. This article is licensed under a Creative Commons Attribution 4.0 International License, which permits use, sharing, adaptation, distribution and reproduction in any medium or format, as long as you give appropriate credit to the original author(s) and the source, provide a link to the Creative Commons licence, and indicate if changes were made. The images or other third party material in this article are included in the article's Creative Commons licence, unless indicated otherwise in a credit line to the material. If material is not included in the article's Creative Commons licence and your intended use is not permitted by statutory regulation or exceeds the permitted use, you will need to obtain permission directly from the copyright holder. To view a copy of this licence, visit http://creativeco mmons.org/licenses/by/4.0/. The Creative Commons Public Domain Dedication waiver (http://creativecommons.org/publicdomain/ zero/1.0/) applies to the data made available in this article, unless otherwise stated in a credit line to the data. 
mortality rate (14-25\%), even higher in patients who are inappropriately treated [1-4]. From 2000 to 2008, the overall incidence rate of anaerobic BSIs was 8.7 per 100,000 annually [3].

Correct identification of anaerobic BSIs is hampered by their slow growth and numerous taxonomic changes. However, a new technique, matrix-assisted laser desorption ionization-time of flight mass spectrometry (MALDI-TOF MS), correctly identified 91.2\% of anaerobic isolates at the species level compared to identification by $16 \mathrm{~S}$ ribosomal RNA (rRNA) sequencing, which is considered the gold standard [5]. As the mortality rate is higher for patients with inadequate antimicrobial therapy [6], MALDI-TOF MS enables a more accurate microbiologic identification of anaerobic BSIs, allowing for appropriate treatment.

Anaerobic organisms are classified as gram-negative bacilli, gram-negative cocci, gram-positive spore-forming bacilli, gram-positive non-spore-forming bacilli, and gram-positive anaerobic cocci (GPAC) $[2,7]$. The majority of anaerobic gram-negative bacilli are Bacteroides spp., with $B$. fragilis isolated most often from clinical infections [8-10]. Clostridium spp. is the main grampositive spore-forming bacilli, with $C$. perfringens as the most frequently identified [11, 12]. However, despite epidemiologic and microbiological studies of anaerobic bacteremia using MALDI-TOF MS and 16S rRNA, the clinical characteristics and infection severity of anaerobic genera remain unclear $[4,10,13]$.

In this study, we retrospectively analyzed anaerobic BSIs identified by MALDI-TOF MS to determine the clinical and microbiological features and patient outcomes based on the anaerobic genera or group. Furthermore, we conducted a multivariate analysis to evaluate the independent risk factors for in-hospital mortality of anaerobic BSIs. The information presented in this study may be helpful to physicians when diagnosing patients with anaerobic BSIs, and for determining appropriate treatment strategies to improve outcomes in patients with anaerobic BSIs.

\section{Methods}

\section{Patients and study design}

This was a single-center retrospective cohort study conducted from January 2016 to December 2020 at the Japanese Red Cross Nagoya Daini Hospital, an 812-bed tertiary medical center in Nagoya, Japan. This research was approved by the Clinical Research Ethics Committee of the Japanese Red Cross Nagoya Daini Hospital (reference no.1483). As this study does not contain any personal information that could lead to the identification of the patient, the requirement for informed consent was waived by the ethics committee. All subjects were over
18 years of age, and patients diagnosed with anaerobic BSI were eligible for inclusion.

\section{Data collection}

Patients' data were collected by reviewing electronic medical records. The primary outcome was in-hospital mortality, defined as all-cause mortality at the hospital after index positive blood cultures.

Demographic data included age, sex, body mass index, and community-onset infection (yes or no). Clinical data included fever, steroid use, underlying diseases, indwelling catheter, and comorbidity severities, recorded according to the Charlson Comorbidity Index (CCI) [14]. Laboratory data were also collected, including the white blood cell count (per $\mu \mathrm{L})$ and creatinine $(\mathrm{mg} / \mathrm{dL})$ and lactate $(\mathrm{mmol} / \mathrm{L})$ levels. The following information were also collected: the Pitt bacteremia score (PBS) for illness severity in the first $24 \mathrm{~h}$ following BSI onset [15], the hospitalization ward, treatments after admission (e.g., vasopressor use, mechanical ventilation, surgical procedures, and appropriate antibiotic prescription), and outcomes (e.g., length of hospital stay and in-hospital mortality). Microbiological data included the likely BSI source, mono- and poly-microorganisms, and the number of bacteremic pathogens (aerobic and anaerobic).

\section{Species identification and antibiotic sensitivity test}

Blood culture samples were incubated for 7 days in the BACT/ALERT 3D instrument (bioMérieux, Marcyl'Étoile, France) per the manufacturer's protocol. BACT/ ALERT FA and FN plus (aerobic and anaerobic) bottles were used for the blood cultures. Subcultures were streaked onto the $\mathrm{BBL}^{\mathrm{TM}} \mathrm{CDC}$ anaerobic $5 \%$ sheep blood agar (BD Biosciences, Franklin Lakes, NJ, USA) from the Centers for Disease Control and Prevention and incubated under anaerobic conditions for 2 days. The incubation period was extended if the bacterial growth was insufficient.

Anaerobic bacteria were identified using the MALDI Biotyper (Bruker Daltonics, Inc., Billerica, MA, USA). Bacterial isolates of single colonies were identified using the plate extraction method as previously described by Theel et al. [16]. MALDI-TOF target plates were inoculated with samples from freshly grown bacterial colonies, overlaid with $1 \mu \mathrm{L}$ of $70 \%$ formic acid (FUJIFILM, Tokyo, Japan), and then overlaid with $1 \mu \mathrm{L}$ matrix ( $\alpha$-cyano4-hydroxycinnamic acid). The bacterial test standard (Bruker Daltonics, Inc.) was used for instrument calibration. Mass spectra were analyzed in an $\mathrm{m} / \mathrm{z}$ range of 2000-20,000. MALDI Biotyper software version 3.0 and the most recent MALDI Biotyper libraries were used for bacterial identification. 
For each anaerobic bacterial strain, two preparations of colony/sample material were analyzed. The Biotyper software compared each sample's mass spectrum to the reference mass spectrum in the database and calculated an arbitrary unit score value between 0 and 3, reflecting similarities between the sample and reference spectrum, then displayed the top ten matching database records. Log scores were interpreted according to the manufacturer's instructions. Log scores of $>2.0$ indicated species identification with high confidence, 1.7-2 indicated low confidence, and $<1.7$ was unreliable identification.

\section{Definitions}

Anaerobic BSI was defined by the isolation of obligate anaerobes from one or more sets of aseptically obtained blood culture bottles. Anaerobic polymicrobial BSI was defined as the simultaneous isolation of anaerobic organisms and one or more other organisms from the blood cultures. BSI was considered clinically relevant when a patient had one or more positive anaerobe blood cultures with clinical evidence consistent with infection (e.g., temperature $>38^{\circ} \mathrm{C}$, elevated serum C-reactive protein concentrations $[>40 \mathrm{mg} / \mathrm{L}]$, or the infection source was definitively identified). Community-onset bacteremia was defined as an infection acquired outside of the hospital setting with a positive blood culture obtained within $48 \mathrm{~h}$ of admission. Nosocomial onset was defined as an infection acquired while being administered treatment within a healthcare setting, or with a positive blood culture obtained more than $48 \mathrm{~h}$ after admission with no evidence of infection at admission. Neutropenia was defined as absolute neutrophil counts of $1000 / \mathrm{mm}^{3}$ or below when bacteremia occurred. An infection focus was defined by the fulfillment of at least two of the following criteria: bacterial isolation from a focal culture, radiologic or clinical signs of focal infection, or focal infection symptoms. If the BSI source could not be specifically attributed, it was classified as a primary BSI. Initial inappropriate therapy was defined as the administration of empiric antimicrobials that were inactive against the isolated microorganisms, including anaerobes and other causative pathogens, or when an appropriate antimicrobial agent was not administered within $24 \mathrm{~h}$ of BSI onset, delaying the administration of antimicrobials. Antibiotic susceptibilities were interpreted according to the Clinical and Laboratory Standards Institute M100 S28 (2018) breakpoints for anaerobes.

\section{Statistical analysis}

SPSS Statistics (version 22; IBM Japan, Tokyo, Japan) was used for statistical analyses. Categorial data and continuous data were expressed as total numbers (percentages) and medians with interquartile ranges (IQRs), respectively. To analyze categorical variables, we used the chi-squared test or Fisher's exact test when appropriate. For continuous variables, we used the Mann-Whitney U test. Logistic regression was used to identify independent risk factors for in-hospital mortality. Known risk factors and factors that were deemed important were included, with or without a low $p$-value. The age, sex, solid tumor, intensive care unit (ICU) stay, Clostridium spp., Bacteroides spp., and GPAC variables had complete datasets, but the initial inappropriate antibiotics dataset was only $97 \%$ complete $(\mathrm{n}=208)$.

\section{Results}

\section{Patients and isolates}

There were 215 patients with anaerobic BSI. Multiple anaerobic organisms were isolated in the blood cultures of 31 out of 215 patients. Therefore, there were 264 total anaerobic BSI episodes (Table 1). In all cases, BSI-causing microorganisms were identified using MALDI-TOF MS. The most frequently isolated anaerobes were Bacteroides spp. $(\mathrm{n}=74)$, followed by gram-positive non-sporeforming bacilli $(\mathrm{n}=57)$, Clostridium spp. $(\mathrm{n}=52)$, GPAC $(\mathrm{n}=27)$, and gram-negative cocci $(\mathrm{n}=7) . B$. fragilis $(\mathrm{n}=35)$ was the most common Bacteroides spp. species, as was Eggerthella lenta $(\mathrm{n}=19)$ for gram-positive nonspore-forming bacilli, C. perfringens $(\mathrm{n}=29)$ for Clostridium spp., and Parvimonas micra (P. micra) $(\mathrm{n}=23)$ for GPAC. Nearly all anaerobic isolates $(90 \%, 237$ out of 264$)$ were identified at the species level; 27 isolates were undetermined (13 anaerobic gram-positive bacilli, 5 anaerobic gram-negative bacilli, 4 Fusobacterium spp., 2 GPAC, 2 Clostridium spp., and 1 Bacteroides spp.).

\section{Patient and infection characteristics}

The median age of anaerobic BSI patients was 76 years $(\mathrm{n}=215$; interquartile range [IQR], 66-84), 56.7\% were male, and community acquisition of anaerobic BSI was 80.9\% (Table 2). The most common comorbidity was a solid tumor (28.8\%), followed by diabetes mellitus (23.7\%), and the median CCI was 5 (IQR, 3-7). Most patients had a fever (68.3\%), and the median lactate level was $2.3 \mathrm{mg} / \mathrm{dL}$ (IQR, 1.4-3.9). The most common focal infections were intra-abdominal (36.7\%), hepatobiliary $(12.1 \%)$, and oropharyngeal (6.5\%); $22.8 \%$ had unknown foci of infection. There were 92 patients (42.8\%) with polymicrobial BSIs (anaerobic microorganisms with other species).

Patients with BSIs caused by Bacteroides spp. and Clostridium spp. often had solid tumors and diabetes mellitus with trans-abdominal and hepatobiliary focal infection sites (Table 2). Patients with GPAC commonly had oropharyngeal (29.6\%) and intra-abdominal (29.6\%) focal infection sites. 
Table 1 Anaerobic microorganisms isolated from bloodstream infections

\begin{tabular}{|c|c|c|c|c|c|}
\hline \multicolumn{3}{|l|}{ Gram negative } & \multicolumn{3}{|l|}{ Gram positive } \\
\hline \multicolumn{3}{|l|}{ Bacilli } & \multicolumn{3}{|l|}{ Spore forming bacilli } \\
\hline Bacteroides & & 74 & Clostridium & & 52 \\
\hline & fragilis & 35 & & perfringens & 29 \\
\hline & thetaiotaomicron & 15 & & ramosum & 5 \\
\hline & vulgatus & 5 & & innocuum & 5 \\
\hline & uniformis & 5 & & clostridioforme & 3 \\
\hline & ovatus & 4 & & paraputrificum & 2 \\
\hline & cellulosilyticus & 3 & & bifermentans & 1 \\
\hline & caccae & 2 & & butyricum & 1 \\
\hline & stercoris & 2 & & cadaveris & 1 \\
\hline & plebeius & 1 & & hathewayi & 1 \\
\hline & pyogenes & 1 & & septicum & 1 \\
\hline & spp & 1 & & symbiosum & 1 \\
\hline \multirow[t]{5}{*}{ Fusobacterium } & & 14 & & spp & 2 \\
\hline & necrophorum & 6 & & & \\
\hline & nucleatum & 2 & Non-spore forming bacillis & & \\
\hline & mortiferum & 2 & Eggerthella lenta & & 19 \\
\hline & spp & 4 & Lactobacillus & & 16 \\
\hline \multirow[t]{3}{*}{ Parabacteroides } & & 5 & & paracasei & 4 \\
\hline & distasonis & 4 & & fermentum & 2 \\
\hline & merdae & 1 & & gasseri & 2 \\
\hline \multirow[t]{4}{*}{ Provotella } & & 2 & & rhamnosus & 2 \\
\hline & buccae & 1 & & salivarius & 2 \\
\hline & melaninogenica & 1 & Actinomyces & & 9 \\
\hline & & & & neuii & 3 \\
\hline Cocci & & & & odontolyticus & 2 \\
\hline \multirow[t]{5}{*}{ Veillonella } & & 7 & & oris & 2 \\
\hline & atypica & 1 & & meyeri & 1 \\
\hline & parvula & 6 & & turicensis & 1 \\
\hline & & & Bifidobacterium & & 6 \\
\hline & & & & longum & 3 \\
\hline Undermined organism & & & & breve & 2 \\
\hline \multirow[t]{16}{*}{ Anaerobic gram-negative bacili } & & 5 & & adolescentis & 1 \\
\hline & & & Cutibacterium & & 6 \\
\hline & & & & acnes & 5 \\
\hline & & & & avidum & 1 \\
\hline & & & Eubacterium limosum & & 2 \\
\hline & & & Solobacterium moorei & & 2 \\
\hline & & & Actinotignum schaalii & & 1 \\
\hline & & & Atopobium parvulum & & 1 \\
\hline & & & Coprobacillus cateniformis & & 1 \\
\hline & & & Cocci & & \\
\hline & & & Parvimonas micra & & 23 \\
\hline & & & Ruminococcus gnavus & & 3 \\
\hline & & & Peptoniphilus indolicus & & 1 \\
\hline & & & Undermined organism & & \\
\hline & & & Anaerobic gram-positive bacili & & 13 \\
\hline & & & Anaerobic gram-positive cocci & & 2 \\
\hline Total & & 107 & Total & & 157 \\
\hline
\end{tabular}


Table 2 Baseline characteristics of patients with anaerobic bloodstream infections

\begin{tabular}{|c|c|c|c|c|c|}
\hline Factor & $\begin{array}{l}\text { All cases } \\
(n=215)\end{array}$ & $\begin{array}{l}\text { Bacteroides spp } \\
(n=60)\end{array}$ & $\begin{array}{l}\text { Non-spore } \\
\text { forming bacillis } \\
(n=57)\end{array}$ & $\begin{array}{l}\text { Clostridium spp } \\
(n=52)\end{array}$ & $\begin{array}{l}\text { GPAC } \\
(n=27)\end{array}$ \\
\hline \multicolumn{6}{|l|}{ Demographics } \\
\hline Age, median (IQR) & $76(66-84)$ & $74.9(67.8-84.3)$ & $74(58-85)$ & $77(69-83)$ & $83(69-86)$ \\
\hline Male sex & $122 / 215(56.7)$ & $33 / 60(55)$ & $31 / 57(54.4)$ & $30 / 52(57.7)$ & $14 / 27(51.9)$ \\
\hline BMI median (IQR) & $20.2(17.8-23.0)$ & $20.4(18.1-22.2)$ & $19.1(17.3-23.0)$ & $20.5(17.7-23.2)$ & $21.1(18.0-23.3)$ \\
\hline Community onset & 174/215 (80.9) & $47 / 60(78.3)$ & $48 / 57(84.2)$ & $38 / 52(73.1)$ & $27 / 32(84.3)$ \\
\hline Nosocomial onset & $41 / 215(19.1)$ & $13 / 60(21.7)$ & 9/57 (15.8) & 14/52 (26.9) & $5 / 32(15.6)$ \\
\hline \multicolumn{6}{|l|}{ Comorbidities } \\
\hline HIV infection & 0 & 0 & 0 & 0 & 0 \\
\hline ESRD on hemodialysis & $13 / 215(6.0)$ & $5 / 60(8.3)$ & $2 / 57(3.5)$ & $3 / 52(5.8)$ & $1 / 27(3.7)$ \\
\hline Diabetes mellitus & $51 / 215(23.7)$ & $13 / 60(21.7)$ & 10/57 (17.5) & 18/52 (34.6) & $6 / 27(22.2)$ \\
\hline Cirrhosis & 4/215 (1.9) & $1 / 60(1.7)$ & $1 / 57(1.8)$ & 2/52 (3.8) & $1 / 27(3.7)$ \\
\hline $\mathrm{CHF}$ & $31 / 215(14)$ & $5 / 60(8.3)$ & 9/57 (15.8) & 10/52 (19.2) & $3 / 27(11.1)$ \\
\hline COPD or severe asthma & $3 / 215(1.4)$ & 0 & $2 / 57(3.5)$ & $1 / 52(1.9)$ & 0 \\
\hline Solid tumor & $62 / 215(28.8)$ & $22 / 60(36.7)$ & $14 / 57(24.6)$ & $13 / 52(25)$ & 8/27 (29.6) \\
\hline Hematological malignancy & $11 / 215(5.1)$ & $3 / 60(5)$ & $2 / 57(3.5)$ & 2/52 (3.8) & $1 / 27(3.7)$ \\
\hline Alcoholism & $4 / 215(1.9)$ & 0 & $1 / 57(1.8)$ & $1 / 52(1.9)$ & $2 / 27(7.4)$ \\
\hline Steroid use & $15 / 215(7.0)$ & $5 / 60(8.3)$ & $6 / 57(10.5)$ & $3 / 52(5.8)$ & $1 / 27(3.7)$ \\
\hline Charlson Comordity Index, median (IQR) & $5(3-7)$ & $5(4-7)$ & $5(2-6)$ & $5(4-7)$ & $5(3-7)$ \\
\hline \multicolumn{6}{|l|}{ Characteristics at presentation } \\
\hline Fever & $147 / 215(68.3)$ & $38 / 60(63.3)$ & $32 / 57(56.1)$ & $39 / 52(75)$ & $22 / 27(81.5)$ \\
\hline Neutropenia (ANC < 1000/mL) & $9 / 215(4.2)$ & $3 / 60(5)$ & $1 / 57(1.8)$ & $3 / 52(5.8)$ & $1 / 27(3.7)$ \\
\hline Creatinine (mg/dL), median (IQR) & $1.0(0.7-1.5)$ & $1.05(0.74-1.62)$ & $0.9(0.6-1.4)$ & $1.2(0.8-1.8)$ & $0.8(0.7-1.0)$ \\
\hline Lactate (mmol/L), median (IQR) & $2.3(1.4-3.9)$ & $2.4(1.4-4.2)$ & $2.1(1.2-3.7)$ & $2.6(1.8-4.6)$ & $2.2(1.7-4.4)$ \\
\hline Central line catheter & $11 / 215(5.1)$ & $3 / 60(5)$ & $5 / 57(8.8)$ & $3 / 52(5.8)$ & 0 \\
\hline Urinary catheter & $3 / 215(1.4)$ & $2 / 60(3.3)$ & 0 & $1 / 52(1.9)$ & 0 \\
\hline \multicolumn{6}{|l|}{ Primary source } \\
\hline Oropharyngeal & $14 / 215(6.5)$ & 0 & 4/57 (7.0) & 0 & $8 / 27(29.6)$ \\
\hline Pneumonia & $8 / 215(3.7)$ & 0 & $3 / 57(5.3)$ & $1 / 52(1.9)$ & $1 / 27(3.7)$ \\
\hline Soft tissue infection & $12 / 215(5.6)$ & $3 / 60(5)$ & $6 / 57(10.5)$ & $3 / 52(5.8)$ & 0 \\
\hline Central venous catheter & $3 / 215(1.4)$ & 0 & $3 / 57(5.3)$ & 0 & 0 \\
\hline Hepatobiliary infection & 26/215 (12.1) & $5 / 60(8.3)$ & $4 / 57(7.0)$ & $13 / 52(25)$ & 0 \\
\hline Intra-abdominal & 79/215 (36.7) & $33 / 60(55)$ & $20 / 57(35.1)$ & 19/52 (36.5) & 8/27 (29.6) \\
\hline Bone and joint & $3 / 215(1.4)$ & $2 / 60(3.3)$ & 0 & 0 & $1 / 27(3.7)$ \\
\hline Endocarditis & $1 / 215(0.5)$ & 0 & 0 & 0 & 0 \\
\hline Urinary tract infection & $9 / 215(4.2)$ & $2 / 60(3.3)$ & $2 / 57(3.5)$ & $1 / 52(1.9)$ & 0 \\
\hline Others & $11 / 215(5.1)$ & $2 / 60(3.3)$ & $1 / 57(1.8)$ & $4 / 52(7.7)$ & $2 / 27(7.4)$ \\
\hline Primary BSI & $49 / 215(22.8)$ & $13 / 60(21.7)$ & $14 / 57(24.6)$ & $11 / 52(21.2)$ & $7 / 27(25.9)$ \\
\hline \multicolumn{6}{|l|}{ Bacteremia } \\
\hline Polymicrobial bacteremia & $92 / 215(42.8)$ & $29 / 60(48.3)$ & $26 / 57(45.6)$ & 27/52 (51.9) & $15 / 27(55.6)$ \\
\hline No. of bacteremic pathogen, median (IQR) & $1(1-2)$ & $1(1-2)$ & $1(1-2)$ & $1(1-2)$ & $1(1-2)$ \\
\hline \multicolumn{6}{|l|}{ Treatment } \\
\hline Portion of initial inappropriate treatment & $36 / 208(17.3)$ & $17 / 59(28.8)$ & $15 / 55(27.2)$ & $5 / 50(10)$ & $5 / 26(19.2)$ \\
\hline Surgical procedure & $51 / 215(23.7)$ & 18/60 (30) & $13 / 57(22.8)$ & $15 / 52(28.8)$ & $7 / 27(25.9)$ \\
\hline ICU care & $35 / 215(16.3)$ & $11 / 60(18.3)$ & $10 / 57(17.5)$ & $12 / 52(23.1)$ & $4 / 27(14.8)$ \\
\hline Vasopressor support & $43 / 215(20.0)$ & $15 / 60(25)$ & $11 / 57(19.3)$ & $15 / 52(28.8)$ & $5 / 27(18.5)$ \\
\hline Ventilatory support & 28/215 (13.0) & $8 / 60(13.3)$ & $8 / 57(14.0)$ & $11 / 52(21.2)$ & $5 / 27(18.5)$ \\
\hline Length of hospital stay, median (IQR) & $20(11-35)$ & $27(17-40)$ & $18(10-42)$ & $21(9-32)$ & $16(11-33)$ \\
\hline Pitt bacteremia score, median (IQR) & $1(0-3)$ & $1.5(0-4)$ & $1(0-3)$ & $2(1-4)$ & $1(0-3)$ \\
\hline
\end{tabular}


Table 2 (continued)

\begin{tabular}{|c|c|c|c|c|c|}
\hline Factor & $\begin{array}{l}\text { All cases } \\
(n=215)\end{array}$ & $\begin{array}{l}\text { Bacteroides spp } \\
(n=60)\end{array}$ & $\begin{array}{l}\text { Non-spore } \\
\text { forming bacillis } \\
(n=57)\end{array}$ & $\begin{array}{l}\text { Clostridium spp } \\
(n=52)\end{array}$ & $\begin{array}{l}\text { GPAC } \\
(n=27)\end{array}$ \\
\hline Mortality & $46 / 215(21.3)$ & $16 / 60(26.7)$ & $11 / 57(19.3)$ & $19 / 52(36.5)$ & $1 / 27(3.7)$ \\
\hline
\end{tabular}

\section{Treatment and outcome}

The in-hospital mortality rate of anaerobic BSI patients was $21.3 \%$. Patients with Clostridium spp. BSIs had the highest mortality rate (36.5\%), whereas those with GPAC BSIs had the lowest (3.7\%). The median hospital stay was 20 days (IQR, 11-35), and inappropriate antibiotics were initially administered to $17.3 \%$ of the patients.

Table 3 presents the characteristics of surviving $(\mathrm{n}=169)$ and deceased $(\mathrm{n}=46)$ patients and predictors for death by univariable analysis. Age, diabetes mellitus, CCI, lactate, Clostridium spp., vasopressor support, and PBS were associated with in-hospital mortality. Fever, oropharyngeal infection, GPAC, surgical procedure, and length of hospital stay were negatively associated with inhospital mortality.

Multivariable analysis showed that age (adjusted odds ratio [aOR], 1.05; 95\% CI, 1.01-1.08), solid tumors (aOR, 2.14; 95\% CI, 1.00-4.57), and Clostridium spp. (aOR, 3.22; 95\% CI, 1.38-7.52) were independent risk factors for in-hospital mortality (Table 4), and GPAC BSIs did not predict a better outcome.

\section{Discussion}

This study determined the most common BSI-causing anaerobes identified by MALDI-TOF MS at a large tertiary center in Japan. Our results indicated that Bacteroides spp. isolates were the most common, followed by anaerobic gram-positive non-spore-forming bacilli and Clostridium spp., consistent with previous studies [3, 4]. We detected a higher frequency of anaerobic gram-positive non-spore-forming bacilli than reported in a study conducted from 1993-2004 [2], and E. lenta was the most common isolate among anaerobic grampositive non-spore-forming bacilli in the current study. Although microbiological and epidemiologic data for anaerobic gram-positive non-spore-forming bacilli are lacking, other studies using MALDI-TOF MS and 16S rRNA gene sequencing support our result that E. lenta is an important anaerobic BSI-causing microorganism [17, 18]. We found 27 GPAC-induced BSI episodes (10.2\% of all cases), which is comparable to the results of Lassmann et al. [2], and P. micra was the most common isolate, identical to the findings by Badri $\mathrm{M}$ et al. [19].

We found that Clostridium spp. BSIs were an independent risk factor for mortality, and the mortality rate was $36.5 \%$. This rate is comparable with the results of
Ngo et al. [3] and Rechner et al. [11], who reported rates of $31 \%$ and $46 \%$, respectively. To identify BSIs caused by Clostridium spp., conventional methods based on biochemical testing are reportedly as reliable as MALDITOF MS, meaning that they may have similar ability to evaluate of BSIs caused by Clostridium spp. [20]. The advantages of the MALDI-TOF MS protocol are rapid identification and reduced reagent usage and labor costs [20, 21]. Rechner et al. [11] also suggested that the high mortality rate for BSIs caused by Clostridium spp. reflected poor health conditions in older and immunocompromised patients. However, Cobo et al. [13] recently reported that a BSI caused by Clostridium spp. was not an independent risk factor for anaerobic BSI mortality, but the study had a smaller sample size than ours $(\mathrm{n}=136)$. C. perfringens is the most common Clostridium spp. and is known to produce a variety of toxins, including lecithinase, a lethal toxin causing massive hemolysis. Although progressive myonecrosis (i.e., gas gangrene) is a life-threatening clostridial infection, there have only been three cases of Clostridium spp.-induced soft tissue infections. Of the 19 fatal cases of BSIs caused by Clostridium spp., 12 were due to hepatobiliary or intra-abdominal infection. Yang et al. [22] reported that nosocomial acquisition, ICU stay, and CCI were associated with a higher mortality rate in patients having a BSI caused by $C$. perfringens. Additionally, it was reported that Clostridium spp. was the contaminant pathogen in $24 \%$ of cases after clinical isolation in blood cultures, $C$. perfringens was the contaminant in $27.3 \%$ of the cases $[22,23]$. Thus, the appropriate initial recognition of the causative Clostridium species is essential for treatment, allowing for patients with BSIs caused by Clostridium spp. to improve.

In contrast to Clostridium spp., the mortality rate of GPAC-induced BSIs was low, at 3.1\%. However, GPACinduced BSIs did not show better outcomes in the multivariable analysis. Earlier studies have shown that the 30-day mortality rates from BSIs caused by GPAC and P. micra, the most common GPAC isolate, were low, at $11 \%$ and $3.8 \%$, respectively $[19,24]$. There were only 46 deaths in this study, which may be an insufficient number to demonstrate a positive association between GPACinduced BSI and outcome. The effects of other GPAC genera, such as Anaerococus spp. and Peptoniphilus spp. (which were not identified in this study), should also be 
Table 3 Comparison of clinical characteristics, and outcomes between surviovors and non-survivors

\begin{tabular}{|c|c|c|c|}
\hline Factor & $\begin{array}{l}\text { Survivors (\%) } \\
(n=169)\end{array}$ & $\begin{array}{l}\text { Deceased (\%) } \\
(n=46)\end{array}$ & $p$ value \\
\hline \multicolumn{4}{|l|}{ Demographics } \\
\hline Age, median (IQR) & $75(64-84)$ & $81(72-86)$ & 0.001 \\
\hline Male sex & $96(56.8)$ & $26(56.5)$ & \\
\hline BMI, median (IQR) & $19.8(18.4-22.1)$ & $19.8(18.5-22.1)$ & \\
\hline Community onset & $140(82.8)$ & $34(73.9)$ & \\
\hline Nosocomial onset & $29(17.2)$ & $12(26.1)$ & \\
\hline \multicolumn{4}{|l|}{ Comorbidities } \\
\hline HIV infection & 0 & 0 & \\
\hline ESRD on hemodialysis & $9(5.3)$ & $4(8.7)$ & \\
\hline Diabetes mellitus & $34(20.1)$ & $17(37.0)$ & 0.02 \\
\hline Cirrhosis & $2(1.2)$ & $2(4.3)$ & \\
\hline $\mathrm{CHF}$ & $22(13.0)$ & $9(19.6)$ & \\
\hline COPD or severe asthma & $3(1.8)$ & 0 & \\
\hline Solid tumor & $44(26.0)$ & $18(39.1)$ & \\
\hline Hematological malignancy & $8(4.7)$ & $3(6.5)$ & \\
\hline Alcoholism & $3(1.8)$ & $1(2.2)$ & \\
\hline Steroid use & $9(5.3)$ & $6(13.0)$ & \\
\hline Charlson Comordity Index & $4(3-6)$ & $6(5-9)$ & $<0.001$ \\
\hline \multicolumn{4}{|l|}{ Characteristics at presentation } \\
\hline Fever & $125(74.0)$ & $22(47.8)$ & $<0.001$ \\
\hline Neutropenia & $5(3.0)$ & $4(8.7)$ & \\
\hline Creatinine, median (IQR) & $1.0(0.7-1.4)$ & $1.2(0.8-2.0)$ & \\
\hline Lactate, median (IQR) & $2.2(1.4-3.3)$ & $3(1.5-7.4)$ & 0.02 \\
\hline Central line catheter & $9(5.3)$ & $2(4.3)$ & \\
\hline Urinary catheter & $3(1.8)$ & 0 & \\
\hline \multicolumn{4}{|l|}{ Primary source } \\
\hline Oropharyngeal & $14(8.3)$ & 0 & 0.04 \\
\hline Soft tissue infection & $9(5.3)$ & $3(6.5)$ & \\
\hline Central venous catheter & $3(1.8)$ & 0 & \\
\hline Hepatobiliary infection & $20(11.8)$ & $6(13.0)$ & \\
\hline Intra-abdominal & $62(36.7)$ & $17(37.0)$ & \\
\hline Bone and joint & $3(1.8)$ & 0 & \\
\hline Urinary tract infection & $8(4.7)$ & $1(2.2)$ & \\
\hline \multicolumn{4}{|l|}{ Bacteremia } \\
\hline Polymicrobial bacteremia & $70(40.2)$ & $22(47.8)$ & \\
\hline No. of bacteremic pathogen, median (IQR) & $1(1-2)$ & $1(1-2)$ & \\
\hline Bacteroides spp & $44(25.3)$ & $16(34.8)$ & \\
\hline Non-spore forming bacillis & $46(26.4)$ & $11(23.9)$ & \\
\hline Clostridium spp & $33(19.0)$ & $19(41.3)$ & 0.009 \\
\hline \multicolumn{4}{|l|}{ Treatment } \\
\hline Initial inappropriate therapy & $30(18.5)$ & $10(21.7)$ & \\
\hline Surgical procedure & $47(27.8)$ & $4(8.7)$ & 0.04 \\
\hline Vasopressor support & $28(16.6)$ & $15(32.6)$ & 0.02 \\
\hline Ventilatory support & $20(11.8)$ & $8(17.4)$ & \\
\hline Length of hospital stay, median (IQR) & $24(15-35)$ & $14(2-30)$ & 0.001 \\
\hline Pitt bacteremia score, median (IQR) & $1(0-3)$ & $3(1-6)$ & $<0.001$ \\
\hline
\end{tabular}


Table 4 Risk factors independently associated with death in patients with anaerobic BSI

\begin{tabular}{llll}
\hline Variables & Adjusted OR & $\begin{array}{l}\text { Multivariable } \\
\mathbf{9 5 \% ~ C l}\end{array}$ & $\boldsymbol{p}$ value \\
\hline Age & 1.05 & $1.01-1.08$ & 0.006 \\
Solid tumor & 2.14 & $1.00-4.57$ & 0.05 \\
Clostridium spp. & 3.22 & $1.38-7.52$ & 0.005 \\
\hline
\end{tabular}

considered. Previous reports have indicated that in-hospital mortality rates of BSIs caused by Anaerococus spp. and Peptoniphilus spp. were low (12\% and 5.8\%, respectively), and those genera should be included to evaluate association with better outcomes of GPAC-induced BSIs [19]. Thus, future studies should clarify if differences among anaerobic genera or groups are associated with anaerobic BSI prognosis.

We also found that patient-related factors, such as age and solid tumors, were independent risk factors for mortality, consistent with previous reports $[13,25,26]$. However, previously reported risk factors for mortality, such as nosocomial infection, ICU admission, and inappropriate initial treatment, were not associated with in-hospital mortality in this study $[13,19,27]$. The small number of deaths or the effects of unmeasured factors associated with mortality may have underestimated certain mortality risk factors in our study.

This study has some limitations. First, since the study was retrospectively designed, some cases may have been missed, and we could only analyze variables already included in the medical records. Second, our study was hospital-based. Therefore, the risk factors for anaerobic BSIs and mortality relative to the general population were not fully investigated. Third, only 46 deaths were recorded, which may not have sufficient power to adjust for all variables associated with anaerobic BSI prognosis. A large-scale, prospective, and multicenter study is required to accurately investigate anaerobic infection epidemiology and mortality risk factors. Finally, we were unable to demonstrate whether the use of MALDI-TOF as opposed to conventional methods changes the treatment strategies and outcomes due to the retrospective study and lack of microbiological data regarding anaerobic pathogens identified by conventional methods.

\section{Conclusions}

This study presents the current trends for anaerobic BSIs identified by MALDI-TOF MS. Bacteroides spp. were the most isolated anaerobes, Clostridium spp. affected the mortality rate of patients with anaerobic BSIs, and both genera often had trans-abdominal and hepatobiliary focal infection sites. These results may assist physicians and clinical microbiology laboratories in the diagnosis and treatment of anaerobic BSIs. Further studies are required to elucidate specific differences in anaerobic genera.

\section{Abbreviations}

aOR: Adjusted odds ratio; B. fragilis: Bacteroides fragilis; BSI: Bloodstream infection; CCl: Charlson Comorbidity Index; Cl: Confidence interval; C. perfringens: Clostridium perfringens; E. lenta: Eggerthella lenta; GPAC: Gram-positive anaerobic cocci; INQ: Interquartile range; MALDI-TOF MS: Matrix-assisted laser desorption ionization-time of flight mass spectrometry; PBS: Pitt bacteremia score; P. micra: Parvimonas micra; rRNA: Ribosomal RNA.

\section{Acknowledgements}

The authors would like to acknowledge all members of the Division of Rheumatology, Chubu Rosai Hospital and Division of General Internal Medicine, Japanese Red Cross Nagoya Daini Hospital for their helpful discussion.

\section{Authors' contributions}

TW and YH designed the protocol and collected the data. TW, YH, and YY analyzed and interpreted the patient data. TW wrote the original draft. TW, YH, YY, WYK, YF, MY, and YN reviewed and edited the manuscript. All authors read and approved the manuscript.

Funding

None.

Availability of data and materials

The datasets for the current study are available from the corresponding author on reasonable request.

\section{Declarations}

Ethics approval and consent to participate

This retrospective research was approved by the Clinical Research Ethics Committee of Japanese Red Cross Nagoya Daini Hospital (reference no.1483). The study data were anonymized before use. As this case series does not contain any personal information that could lead to patient identification, the requirement for informed consent was waived by the ethics committee.

\section{Consent for publication}

Not applicable.

\section{Competing interests}

The authors declare that they have no competing interests.

\section{Author details}

${ }^{1}$ Division of Rheumatology, Chubu Rosai Hospital, 2-10-15, Komei-cho, Minato-ku, Nagoya, Aichi 455-8530, Japan. ${ }^{2}$ Division of Clinical Laboratory, Japanese Red Cross Nagoya Daini Hospital, Nagoya, Aichi 466-8650, Japan. ${ }^{3}$ Division of General Internal Medicine, Japanese Red Cross Nagoya Daini Hospital, Nagoya, Aichi 466-8650, Japan. ${ }^{4}$ Division of Nephrology, Chubu Rosai Hospital, 2-10-15, Komei-cho, Minato-ku, Nagoya, Aichi 455-8530, Japan.

Received: 29 March 2021 Accepted: 2 June 2021

Published online: 09 June 2021

References

1. Salonen JH, Eerola E, Meurman O. Clinical significance and outcome of anaerobic bacteremia. Clin Infect Dis. 1998;26(6):1413-7.

2. Lassmann B, Gustafson DR, Wood CM, Rosenblatt JE. Reemergence of anaerobic bacteremia. Clin Infect Dis. 2007;44(7):895-900.

3. Ngo JT, Parkins MD, Gregson DB, Pitout JD, Ross T, Church DL, et al. Population-based assessment of the incidence, risk factors, and outcomes of anaerobic bloodstream infections. Infection. 2013;41(1):41-8. 
4. De Keukeleire S, Wybo I, Naessens A, Echahidi F, Van der Beken M, Vandoorslaer K, et al. Anaerobic bacteraemia: a 10-year retrospective epidemiological survey. Anaerobe. 2016;39:54-9.

5. Garner O, Mochon A, Branda J, Burnham CA, Bythrow M, Ferraro M, et al. Multi-centre evaluation of mass spectrometric identification of anaerobic bacteria using the VITEK ${ }^{\circledR}$ MS system. Clin Microbiol Infect. 2014;20(4):335-9.

6. Zahar JR, Farhat H, Chachaty E, Meshaka P, Antoun S, Nitenberg G. Incidence and clinical significance of anaerobic bacteraemia in cancer patients: a 6-year retrospective study. Clin Microbiol Infect. 2005;11(9):724-9.

7. Fenner L, Widmer AF, Straub C, Frei R. Is the incidence of anaerobic bacteremia decreasing? Analysis of 114,000 blood cultures over a ten-year period. J Clin Microbiol. 2008;46(7):2432-4.

8. Redondo MC, Arbo MD, Grindlinger J, Snydman DR. Attributable mortality of bacteremia associated with the Bacteroides fragilis group. Clin Infect Dis. 1995;20(6):1492-6.

9. Cheng CW, Lin HS, Ye JJ, Yang CC, Chiang PC, Wu TS, et al. Clinical significance of and outcomes for Bacteroides fragilis bacteremia. J Microbiol Immunol Infect. 2009:42(3):243-50.

10. Dumont Y, Bonzon L, Michon AL, Carriere C, Didelot MN, Laurens C, et al. Epidemiology and microbiological features of anaerobic bacteremia in two French University hospitals. Anaerobe. 2020;64:102207.

11. Rechner PM, Agger WA, Mruz K, Cogbill TH. Clinical features of clostridial bacteremia: a review from a rural area. Clin Infect Dis. 2001;33(3):349-53.

12. Leal J, Gregson DB, Ross T, Church DL, Laupland KB. Epidemiology of Clostridium species bacteremia in Calgary, Canada, 2000-2006. J Infect. 2008;57(3):198-203.

13. Cobo F, Aliaga L, Expósito-Ruiz M, Navarro-Marí JM. Anaerobic bacteraemia: a score predicting mortality. Anaerobe. 2020;64:102219.

14. Charlson ME, Pompei P, Ales KL, MacKenzie CR. A new method of classifying prognostic comorbidity in longitudinal studies: development and validation. J Chronic Dis. 1987:40(5):373-83.

15. Paterson DL, Ko WC, Von Gottberg A, Mohapatra S, Casellas JM, Goossens $\mathrm{H}$, et al. International prospective study of Klebsiella pneumoniae bacteremia: implications of extended-spectrum beta-lactamase production in nosocomial Infections. Ann Intern Med. 2004;140(1):26-32.

16. Theel ES, Schmitt BH, Hall L, Cunningham SA, Walchak RC, Patel R, et al. Formic acid-based direct, on-plate testing of yeast and Corynebacterium species by Bruker Biotyper matrix-assisted laser desorption ionizationtime of flight mass spectrometry. J Clin Microbiol. 2012;50(9):3093-5.

17. Gardiner BJ, Tai AY, Kotsanas D, Francis MJ, Roberts SA, Ballard SA, et al. Clinical and microbiological characteristics of Eggerthella lenta bacteremia. J Clin Microbiol. 2015;53(2):626-35.
18. Ugarte-Torres A, Gillrie MR, Griener TP, Church DL. Eggerthella lenta bloodstream infections are associated with increased mortality following empiric piperacillin-tazobactam (TZP) monotherapy: a Population-based Cohort Study. Clin Infect Dis. 2018;67(2):221-8.

19. Badri M, Nilson B, Ragnarsson S, Senneby E, Rasmussen M. Clinical and microbiological features of bacteraemia with Gram-positive anaerobic cocci: a population-based retrospective study. Clin Microbiol Infect. 2019;25(6):760.e1-760.e6.

20. Jamal WY, Shahin M, Rotimi VO. Comparison of two matrix-assisted laser desorption/ionization-time of flight (MALDI-TOF) mass spectrometry methods and API 20AN for identification of clinically relevant anaerobic bacteria. J Med Microbiol. 2013;62:540-4.

21. Tan KE, Ellis BC, Lee R, Stamper PD, Zhang SX, Carroll KC. Prospective evaluation of a matrix-assisted laser desorption ionization-time of flight mass spectrometry system in a hospital clinical microbiology laboratory for identification of bacteria and yeasts: a bench-by-bench study for assessing the impact on time to identification and cost-effectiveness. J Clin Microbiol. 2012;50:3301-8.

22. Yang CC, Hsu PC, Chang HJ, Cheng CW, Lee MH. Clinical significance and outcomes of Clostridium perfringens bacteremia-a 10-year experience at a tertiary care hospital. Int J Infect Dis. 2013;17(11):e955-60.

23. Pien BC, Sundaram P, Raoof N, Costa SF, Mirrett S, Woods CW, et al. The clinical and prognostic importance of positive blood cultures in adults. Am J Med. 2010;123(9):819-28.

24. Watanabe T, Hara Y, Yoshimi Y, Fujita Y, Yokoe M, Noguchi Y. Clinical characteristics of bloodstream infection by Parvimonas micra: retrospective case series and literature review. BMC Infect Dis. 2020;20(1):578.

25. Hung MN, Chen SY, Wang JL, Chang SC, Hsueh PR, Liao CH, et al. Community-acquired anaerobic bacteremia in adults: one-year experience in a medical center. J Microbiol Immunol Infect. 2005;38(6):436-43.

26. Umemura T, Hamada Y, Yamagishi Y, Suematsu H, Mikamo H. Clinical characteristics associated with mortality of patients with anaerobic bacteremia. Anareobe. 2016:39:45-50.

27. Kim J, Lee Y, Park Y, Kim M, Choi JY, Yong D, et al. Anaerobic bacteremia: impact of inappropriate therapy on mortality. Infect Chemother. 2016:48(2):91-8.

\section{Publisher's Note}

Springer Nature remains neutral with regard to jurisdictional claims in published maps and institutional affiliations.
Ready to submit your research? Choose BMC and benefit from:

- fast, convenient online submission

- thorough peer review by experienced researchers in your field

- rapid publication on acceptance

- support for research data, including large and complex data types

- gold Open Access which fosters wider collaboration and increased citations

- maximum visibility for your research: over $100 \mathrm{M}$ website views per year

At BMC, research is always in progress.

Learn more biomedcentral.com/submissions 